\title{
LES ANTOCHA (IMAGOS) DU SUD DU LIBAN [DIPTERA, LIMONIIDAE]
}

\author{
par A. G. B. THOMAS 1 et A. DIA ${ }^{2}$
}

Description illustrée (antenne, aile, terminalia) des imagos mâle et femelle d'Antocha (s. str.) phoenicia n. sp., proche d'A. vitripennis (Meigen, 1830) ; redescription des imagos ot ot o d'A. (s. str.) libanotica Lackschewitz, 1939.

\section{The imagos of Antocha from the South of Lebanon (Diptera, Limoniidae)}

Illustrated description (antenna, wing, terminalia) of the male and female imagos of Antocha (s. str.) phoenica n. sp., nearly allied to A. vitripennis (Meigen, 1830) ; redescription of the male and female imagos of $A$. (s. str.) libanotica Lackschewitz, 1939.

\section{1. - INTRODUCTION}

Les stades immatures des Limoniidae du genre Antocha OstenSacken (= Taphrophila Rondani) comptent parmi les Diptères les plus franchement aquatiques et les plus fréquents en eau courante. Leur écologie et leur biologie sont néanmoins fort peu connues.

Actuellement, deux espèces seullement sont citées avec certitude d'Europe occidentale : Antocha vitripennis (Meigen, 1830) et A. fulvescens Lackschewitz, 1939.

La faune orientale (Inde en particulier) est beaucoup plus diversifiée et compte, pour le moins, plusieurs dizaines d'espèces.

Du Liban, à notre connaissance, seule A. libanotica Lackschewitz, 1939 a été signalée. La prospection de plusieurs cours d'eau de deux bassins hydrographiques du Sud de ce pays par l'un de nous (A. Dia, Université de Beyrouth) a permis la récolte de spécimens de deux espèces: A. libanotica et $A$. phoenicia n. sp. Le matériel est conservé en alcool à $70^{\circ}$.

1. Laboratoire d'Hydrobiologie, ERA 702 du CNRS, Université Paul-Sabatier, 118, route de Narbonne, 31062 Toulouse Cedex.

2. Département Sciences Naturelles, Université Libanaise, Faculté des Sciences, Hadeth-Beyrouth, Liban.

Ce travail a été subventionné par le CNRS hibanais. 


\section{2. - ANTOCHA LIBANOTICA LACKSCHEWITZ, 1939}

\section{DESCRIPTION}

\section{Mâle}

Taille. Longueur de l'aile : 5,7 à $6,6 \mathrm{~mm}$.

Tête

Brun foncé, un peu plus claire ventralement. Trompe jaune, excepté les labelles, en partie bruns. Palpes bruns sauf l'apex, blanc grisâtre. Antennes (fig. 11) brun soutenu à 16 articles, ceux du flagelle assez fusiformes et porteurs d'un manchon de fines soies périphériques; quelques soies plus fortes, verticillées, sont insérées sur les articles 7, $9,11,13,15$ et 16 en particulier, alors qu'elles sont absentes sur les

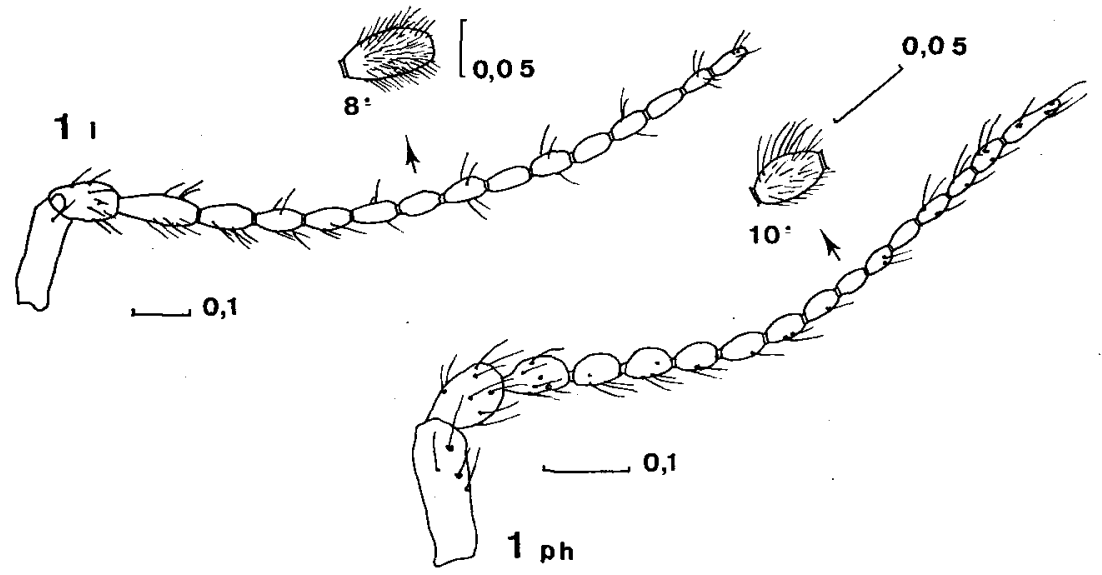

0,5

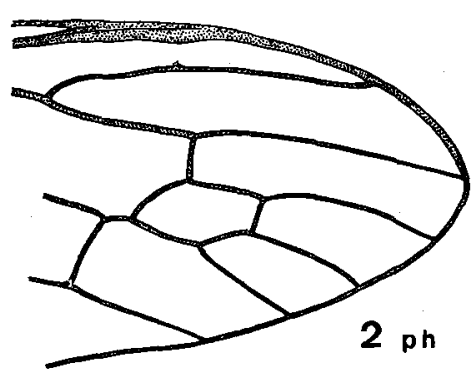

0,5

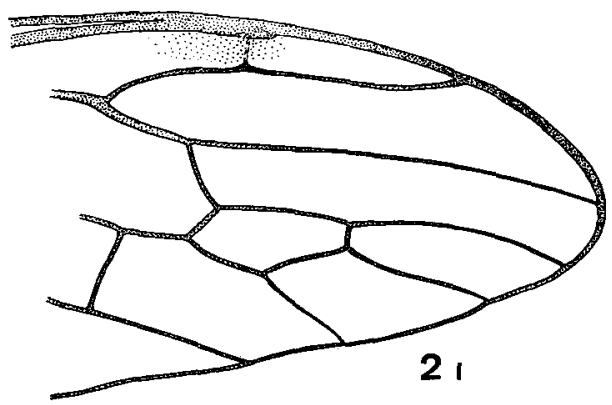

Planche I : FIg. 1 et 2. - Antocha libanotica Lackschewitz (l) et A. phoenicia $\mathrm{n}$. sp. (ph.). Echelle en mm.

FIG. 1 : antenne $\left(\sigma^{*}\right)$. FIG. 2 : rágion apicale de l'aile $\left(0^{*}\right)$. 
articles intercalaires 8, 10, 12 et 14. Rapport de la longueur du premier article du flagelle sur son diamètre : 2,9. Scape brun jaunâtre sale.

\section{Thorax}

Prescutum presque entièrement recouvert par trois larges bandes brun moyen : une médiane et deux latérales séparées par une très fine ligne claire à peine visible. Epaulements antérieurs et bords latéraux du prescutum plus clairs. Scutellum brun grisâtre clair et sale. Metanotum et pleures brun grisâtre.

Ailes relativement étroites correspondant à la fig. $4 \mathrm{~b}$ de Savtshenko (1981). Membrane alaire nettement grisâtre, moins hyaline que celle d'A. vitripennis. Nervation brune, quelque peu jaunâtre dans la région apicale et antérieure. Tache ptérostigmatique et nervure transverse rq peu: marquées mais visibles (fig. 21 ). Cellule discale fermée.

Balanciers grisâtres à massue presque sphérique et tige longue et finc.

Coxas grisâtre sale. Pattes brun moyen assez uniforme à l'exception de la région proximale des fémurs, jaunâtre.

\section{Abdomen}

Brun moyen, avec toutefois les segments 6 à 8 (en particulier le $7^{\mathrm{c}}$ ) nettement plus foncés, brun noirâtre. Pleures grisâtres.

Genitalia jaune foncé à peine embruni, déjà dessinés (en vues dorsalc et ventrale) par Lackschewitz (1939), Lackschewitz et Pagast (1942) et Savtshenko (1981). Epandrium court, très large et formant deux. fortes saillies latérales ovales, de part et d'autre d'une dépression médiane; son bord antérieur n'est cependant pas échancré au milieu. 9: sternite très apparent, triangulaire. Basistyles fortement incurvés dorsalement près de leur base (fig. 3 1). Appendices externes des dististyles en crochets plutôt forts, régulièrement recourbés, assez affinés à l'extrémité (fig. 41). De chaque côté, trois pointes aiguës : la plus interne est solidaire du pénis, les deux autres constituent l'apex d'un large paramère, fortement chitinisé et très visible. Pénis longuement recourbé à angle droit.

\section{Femelle}

Taille. Longueur de l'aile : 5,5 à $7,0 \mathrm{~mm}$.

Coloration comparable à celle du $\hat{o}$ mais thorax un peu plus clair et abdomen dépourvu d'anneau foncé dans la région postérieứe : la tête est la partie la plus foncée.

Pattes plus épaisses et un peu plus claires que chez le 8 .

Ovipositeur : cerques et valves hypogynales longs (fig. $5 \mathrm{l}$ ). 


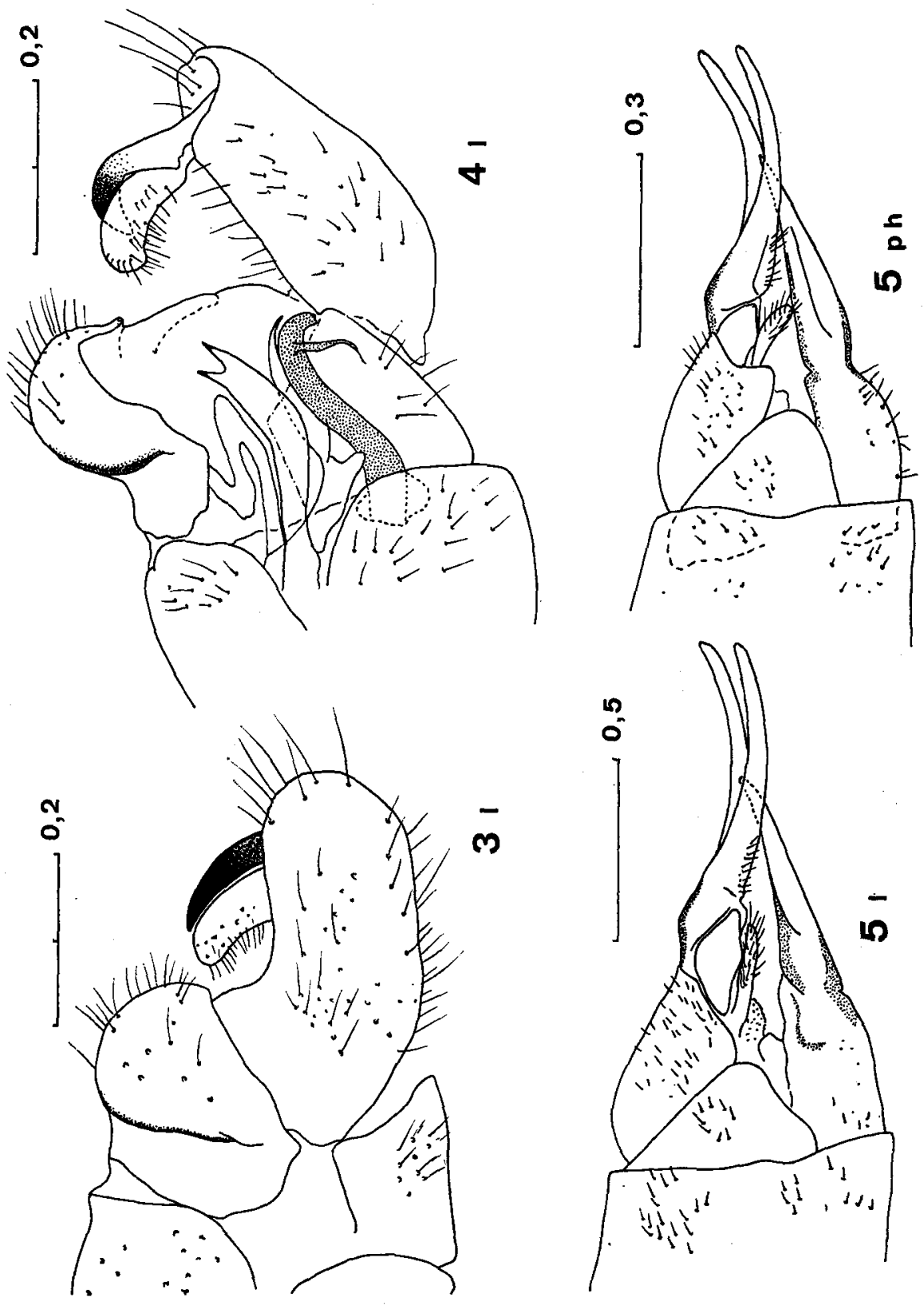

Planche II : Fig. 3 à 5. - Echelle en mm.

Fic. 3: genitalia $\delta^{*}$ d'A. libanotica Lackschewitz en vue latérale. Fig. $4:$ id. en vue latéro-ventrale, demi-forceps gauche retiré. Fig. 5 : terminalia 9 d'A. libanotica (l) et d'A. phoenicia n. sp. (ph.) en vue latérale. 
MATÉRIEl EXAMiné (10 imagos : 6 s, 4 \%)

Sud-Ouest du Liban, entre Saïda et Beyrouth (A. Dia leg.); Nabaa = source.

Bassin du Nahr el Aouali : Nabaa Aazibi au Nord de Jezzine (990 m)

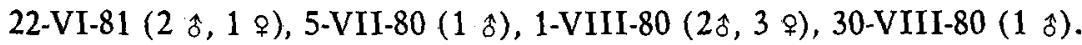

\section{3. - ANTOCHA PHOENICIA THOMAS ET DIA N. SP.}

\section{DESCRIPTION}

Plus claire et plus petite que la précédente, cette espèce est assez proche d'A. vitripennis (Meigen).

\section{Mâle}

Taille. Longueur de l'aile : 4,5 à $6,3 \mathrm{~mm}$.

Tête

Brune dorsalement; trompe jaunâtre à labelles à peine plus foncés. Palpes brun grisâtre sale, de même que la moitié distale des antennes à 16 articles. Région proximale de ces dernières jaunâtre, en particulier le scape. Le $16^{\mathrm{e}}$ article (fig. $1 \mathrm{ph}$ ) est long alors que la plupart des autres et le pédicelle sont plutôt gros et courts (rapport longueur/diamètre du $1^{\text {er }}$ article du flagelle: 1,8 ). Un feutrage de soies claires, net seulement sur la face antérieure des antennes; des soies noires plus grandes, verticillées, visibles surtout sur les articles 13 à 16 .

\section{Thorax}

Préscutum recouvert jusqu'à la suture thoracique - ou presque, selon les individus - par une large bande médiane brune; deux larges bandes latérales de même couleur sont contiguës à la précédente ou même fusionnées avec elle et se prolongent jusqu'au scutellum, grisâtre; régions antérolatérales du préscutum jaunâtres. Scutum foncé latéralement et clair au centre. Metatonum brun soutenu. Pleures brunes.

Ailes plus claires que celles d'A. libanotica et relativement hyalines. Les nervures sont jaune brunâtre jusqu'aux radiales incluses, puis plus foncées vers l'arrière, en particulier la cubitale. Les ailes sont, en fait, voisines de celles d'A. vitripennis, avec la nervure transverse rq peu ou pas visible et pas de tache ptérostigmatique (fig. $2 \mathrm{ph}$ ). Le secteur radial est plus court que chez $A$. libanotica et, au contraire, le segment compris entre l'apex de Rs et la transverse rm bien plus long; la cellule discale, fenmée, est aussi plus courte et plus étroite. 


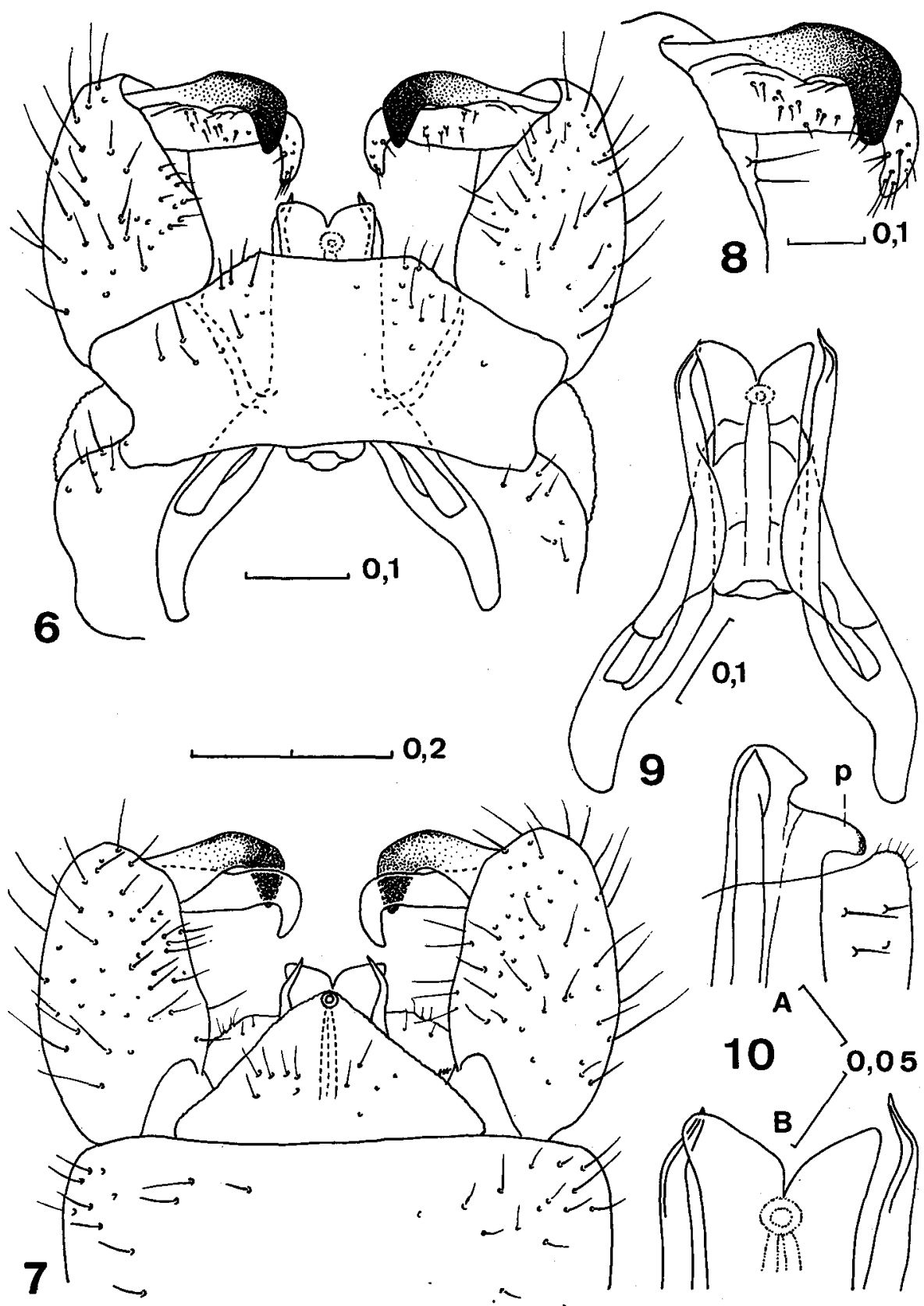

Planche III : Fig. 6 à 10. - Genitalia ơ d'A. phoenicia n. sp. Echelle en mm.

Fig. 6 et 7 : ensemble en vues donsale (6) et ventrale (7). Fig. 8 à 10 : dististyle droit (8), édéage (9) et son apex $(10 \mathrm{~B})$ en vue dorsale; cet apex en vue latérale $(10 \mathrm{~A}) . \mathrm{p}=$ pénis. 
Balanciers à tige jaunâtre et massue blanc grisâtre.

Pattes à pilosité courte et fine. Fémurs jaune brunâtre, le reste un peu plus foncé. Coxas très claires, jaunâtres.

Abdomen

Jaunâtre - les premiers segments parfois même blanchâtres - un pev embruni dorsalement ; pleures blanchâtres. $7^{\mathrm{e}}$ et $8^{\mathrm{e}}$ sternites brun foncé.

Genitalia assez voisins de ceux d'A. vitripennis. Les crochets des dististyles sont cependant plus courts, plus épais et moins pointus ( $f i g$. 6 et $8 \mathrm{ph}$ ). Les paramères aussi sont moins régulièrement incurvés et aigus, mais au contraire aplatis et nettement pliés près de l'apex (fig. 9 et $10 \mathrm{ph}$ ).

Dististyles et paramères rappellent assez ceux d'A. hirtipes Savtshenko, 1971 (Transcaucasie). Mais les genitalia diffèrent par d'autres caractères. D'autre part, la pilosité des pattes est normale, donc très réduite par rapport à celle d'A. hirtipes.

\section{Femelle}

Taille. Longueur de l'aile : 4,7 à $6,9 \mathrm{~mm}$.

Coloration assez comparable à celle du ô sauf :

- scutum plus contrasté, avec une aire antérieure médiane, brun foncé. plus courte ;

- tache distale de l'abdomen beaucoup plus étroite.

Ovipositeur. Les valves hypogynales et surtout les cerques, longs et fins (fig. $5 \mathrm{ph}$ ), éloignent nettement cette espèce d'A. turkestanica De Meijere, 1921 et d'A. Lindneri (Nielsen, 1963) : voir Nielsen (t.c) fig. 3, et fig. 4 s. n. afghana Nielsen, 1952.

Matériel examiné ( 265 imagos : 182 ô et 83 q).

Sud-Ouest du Liban, entre Saïda et Beyrouth (A. Dia leg.) ; Nabaa = source.

- Bassin du Nahr el Aouali : pont du Jdaîdet ech Chouf (710 m)

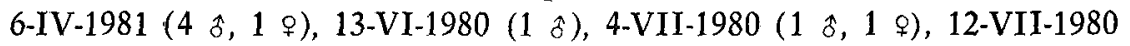
(1 f), 29-VIII-1980 (15 ô, 2 c), 8-XI-1980 (3 ô); Jisr Bisri (380 m) 11-IX1980 (1 $\hat{\delta}, 4$ \&); amont de la centrale hydroélectrique de l'Aouali (230 m) 14-V-1981 (1 ô ), 21-VI-1980 (1 ô), 11-IX-1980 (2 ô); Nabaa Mourched, près d'El Moukhtâra $(800 \mathrm{~m})$ 8-V-1981 (5 $\delta, 1$ q $)$.

- Eassin du Nahr ed Damour: Jisr el Quâdi (260 m) 20-IV-1981

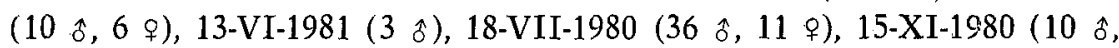
2 f); Jisr ed Damour en aval de sa confluence avec le Nahr el Hammam $(40 \mathrm{~m})$ 24-IV-1981 (12 今, 7 ㅇ), 5-VI-1981 (1 1 ), 22-VII-1980 (27 f, 
30 ९), 24-IX-1980 (9 1 , 2 ) ; affluent temporaire Ouâdi el Ghâboun (confluent à $260 \mathrm{~m}$ ) 20-IV-1981 (1 $\delta)$; affluent Nahr el Hammam (45 m) 21-II-1981 (1 ô), 21-IV-1981 (14 8,8 \%), 22-VII-1980 (22 is dont l'holo-

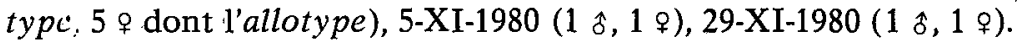

\section{4. - REPARTITION DES DEUX ESPECES}

Nos récoltes ont été échelonnées sur tous les mois de l'année. Dans la. région prospectée, il apparaît nettement que :

- A. phoenicia est beaucoup plus fréquente et abondante qu'A. libanotica ;

- La période de vol d'A. phoenicia - comme celle d'A. vitripennis - est très étalée (capture la plus précoce : 21 février, la plus tardive : 29 novembre) alors que celle d'A. libanotica parait limitée à l'été ( 22 juin au 30 août pour notre matériel, 3 au 10 juin pour le matériel étudié par Lackschewitz).

En outre, A. libanotica semble être une espèce de moyenne montagne ( $990 \mathrm{~m}$ pour nos récoltes, station la plus élevée parmi toutes celles prospectées ; $1400 \mathrm{~m}$ pour la série type, provenant de Bcharré) alors qu' A. phoenicia est surtout rencontrée en basse altitude. Alexander (1975) cite des captures d'A. libanotica en Iran du 18 mai au 21 septembre et jusqu'à $2200 \mathrm{~m}$ d'altitude (F. Schmid leg.).

\section{TRAVAUX CITES}

AleXANDer (C. P.). 1975. - New or little-known Crane Flies from Iran. IV (Diptera: Tipulidae). Jl N.Y. ent. Soc., 83 (2) : 129-138.

LACKSCHEWITZ (P.). 1939 (1940). - Die paläarktischen Rhamphidiinen und Eriopterinen des Wiener Naturhistorischen Museums. Annln naturh. Mus. Wien, $50: 1-67+3 \mathrm{pl}$.

Lackschewitz (P.) et Pagast (F.). 1942. - Limoniidae. In Lindner E. : Die Fliegen der Palaearktischen Region. $145:$ 33-64 + pl. 9-13.

MEIJERE (J. C. H. De). 1921. - Studien über palaearktische, vorwiegend holländische, Limnobiiden, insbesondere über ihre Kopulationsorgane. Tijdschr. Ent., 64: $54-118+8 \mathrm{pl}$.

Nielsen (P.). 1962. - The $3^{\text {rd }}$ Danish expedition to Central Asia. Zoological Results 30. Nematocera (Insecta) from Afghanistan. Vidensk. Meddr dansk naturh. Foren., $124:$ 165-169.

NiELSEN (P.). 1963. - Records and descriptions of Nematocera from Afghanistan (Contribution à l'étude de la faune d'Afighanistan, $\mathrm{n}^{\circ}$ 79). Stuttg. Beitr. Naturk., 118 (3) : 1-8.

Savtshenko (E. N.). 1971. - [New palaearctic species of Limoniid-Flies (Diptera, Limoniidae) II. Genus Antocha O.-S.]. Dopov. Akad. Nauk Ukr. RSR (Ser. $B$ ), $\mathrm{n}^{\circ} 9: 855-857$ (en russe).

SAVTSHENKo (E. N.). 1981. - [Three new species of Limoniid-Flies (Diptera, Limoniidae) from USSR.] Vest. Zoologii, Kiev, $1: 22-29$ (en russe). 